\title{
A Priori Detection of Zeno Behavior in Communication Networks Modeled as Hybrid Systems
}

\author{
Alessandro Abate, Aaron D. Ames and Shankar Sastry \\ Department of Electrical Engineering and Computer Sciences \\ University of California at Berkeley \\ Berkeley, CA 94720 \\ \{aabate, adames, sastry@eecs.berkeley.edu\}
}

\begin{abstract}
In this paper, we show that the sufficient conditions for the existence of Zeno behavior in hybrid systems derived in [3] correctly predict such executions in a modeling instance of the fluid-flow approximation of the TCP-like protocol for wireless communication networks.
\end{abstract}

\section{INTRODUCTION}

Hybrid systems are systems that display both continuous and discrete behavior, and so are endowed with powerful modeling capabilities. Unfortunately, the yin to this yang is that hybrid models may exhibit non robust and pathological behaviors due to their structural interconnections. Zeno behavior provides a notorious, and peculiar, example of this; its existence can be disruptive to both analysis and simulation. This motivates the need to understand Zeno behavior and, specifically, detect it a priori.

The literature on Zeno behavior can be categorized within three general areas: analysis, elimination (or regularization) and detection (either sufficient or necessary conditions for the existence of Zeno behavior). The first area studies the dynamical properties associated with this phenomenon, its relation to real world systems, and the computational problems it arises. The second area attempts to understand how to modify a hybrid model in order to guarantee the absence of such a behavior (see, for instance, [4], [7], [11]). The final area, providing necessary and/or sufficient conditions for the existence of Zeno behavior, has drawn some interest in recent years; while the Literature has results on necessary conditions, which are based on the interconnections between the domains of the system, we focus here on sufficient conditions, which focus on the structure of the vector fields.

In [3], the first—as far as the authors are aware—sufficient conditions for the existence of Zeno behavior in hybrid systems with non-trivial (or non-constant) dynamics were provided; there, a simple class of hybrid systems with simple dynamics, termed diagonal first quadrant (DFQ) hybrid systems was studied. These results were generalized in [6] to include arbitrary nonlinear dynamics through the use of Lyapunov-type techniques.

This work utilizes the framework of hybrid systems to model Communication Networks that employ the Transmission Control Protocol (TCP). These complex models of

This research is supported by the National Science Foundation (NSF award number CCR-0225610) and the grant DAAD19-03-1-0373. networks, based on fluid-flow approximations, are naturally discretized into systems that display both discrete and continuous behavior, i.e., hybrid systems. The use of this paradigm can further our understanding of the performance of these algorithms. In particular, this paper presents a real world application of the sufficient conditions given in [3] to a modeling instance of Zeno behavior in TCP networks. That is, we are able to detect a priori the existence of Zeno behavior in a hybrid system model of these systems.

This paper is structured as follows: after an introduction to the classical framework for hybrid systems (Sec. II), and a brief summary of the sufficient conditions for Zeno behavior presented in [3] (Sec. III), the problem setup is established (Sec. IV); a fluid-flow model for the Reno-type TCP is presented in the wired and wireless cases, Furthermore, a simple topology is introduced and it is demonstrated how a network with this topology can be naturally modeled as a hybrid system. We begin by performing a qualitative analysis of the hybrid trajectories of the resulting hybrid system. The existence of Zeno trajectories is then mathematically established for a simple configuration; the sufficient conditions for the existence of Zeno trajectories [3] are utilized (Sec. V) after properly transforming the model to a DFQ hybrid system. Finally, possible extensions of the methods utilized in the paper are discussed (Sec. VI).

\section{The Setting: Hybrid Dynamical Systems}

Throughout the paper, we will utilize the classical framework for deterministic hybrid systems [14]. Let us start by formally defining a hybrid system, its execution and a dynamical property that will be under study.

Definition 1: Define a hybrid system as a tuple

$$
\mathscr{H}=(Q, E, D, G, R, F),
$$

where

- $Q=\{1, \ldots, m\} \subset \mathbb{Z}$ is a finite set of discrete states,

- $E \subset Q \times Q$ is a set of edges which define the connections between states, where for $e=(i, j) \in E$ we denote its source by $\mathfrak{s}(e)=i$ and its target by $\mathfrak{t}(e)=j$,

- $D=\left\{D_{i}\right\}_{i \in Q}$ is a set of domains, where $D_{i}$ is a compact subset of $\mathbb{R}^{n}$, 
- $G=\left\{G_{e}\right\}_{e \in E}$ is a set of guards, where $G_{e} \subseteq D_{\mathfrak{s}(e)}$,

- $R=\left\{R_{e}\right\}_{e \in E}$ is a set of reset maps, where $R_{e}: G_{e} \subseteq$ $D_{\mathfrak{s}(e)} \rightarrow D_{\mathfrak{t}(e)}$ are smooth maps,

- $F=\left\{f_{i}\right\}_{i \in Q}$ is a set of vector fields such that $f_{i}$ is Lipschitz on $\mathbb{R}^{n}$; the solution to the ODE specified by $f_{i}$ with initial condition $x_{0} \in D_{i}$ at time $t_{0}$ is denoted by $x_{i}(t)$, where $x_{i}\left(t_{0}\right)=x_{0}$.

Executions. An execution or hybrid trajectory ${ }^{1}$ of the hybrid system $\mathscr{H}$ is a tuple:

$$
\chi=(\tau, \xi, \eta)
$$

where

- $\tau=\left\{\tau_{i}\right\}_{i \in \mathbb{N}}$ with $\tau_{0}=0 \leq \tau_{1} \leq \cdots \leq \tau_{j} \leq \cdots$ is a hybrid time sequence or a sequence of switching times,

- $\xi=\left\{\xi_{i}\right\}_{i \in \mathbb{N}}$ with $\xi_{i} \in \bigcup_{i \in Q} D_{i}$ is a sequence of initial conditions,

- $\eta=\left\{\eta_{i}\right\}_{i \in \mathbb{N}}$ with $\eta_{i} \in E$ is a hybrid edge sequence.

Additionally, we require that $\chi=(\tau, \xi, \eta)$ must satisfy the conditions that for $i \in \mathbb{N}$,

$$
\begin{aligned}
\xi_{i} & =x_{\mathfrak{s}\left(\eta_{i}\right)}\left(\tau_{i}\right) \\
\tau_{i+1} & =\min \left\{t \geq \tau_{i}: x_{\mathfrak{s}\left(\eta_{i}\right)}(t) \in G_{\eta_{i}}\right\} \\
\mathfrak{s}\left(\eta_{i+1}\right) & =\mathfrak{t}\left(\eta_{i}\right) \\
\xi_{i+1} & =R_{\eta_{i}}\left(x_{\mathfrak{s}\left(\eta_{i}\right)}\left(\tau_{i+1}\right)\right)
\end{aligned}
$$

We finally require that $x_{\mathfrak{s}\left(\eta_{i}\right)}(t) \in D_{\mathfrak{s}\left(\eta_{i}\right)}$ for all $t \in$ $\left[\tau_{i}, \tau_{i+1}\right]$, which is quite a natural assumption.

Hybrid systems display a phenomenon which does not occur in dynamical systems: Zeno behavior. In simple terms, Zeno behavior occurs when there is a hybrid trajectory that takes an infinite amount of discrete transitions in a finite amount of time. More precisely, we give the following definition:

Definition 2: A hybrid system $\mathscr{H}$ is Zeno if for some execution $\chi$ of $\mathscr{H}$ there exists a finite constant $\tau_{\infty}$ (called the Zeno time) such that

$$
\lim _{i \rightarrow \infty} \tau_{i}=\sum_{i=0}^{\infty}\left(\tau_{i+1}-\tau_{i}\right)=\tau_{\infty}
$$

The execution $\chi$ is called a Zeno execution.

Zeno behaviors. The definition of a Zeno execution results in qualitatively different types of Zeno behavior: the two fundamental kinds ${ }^{2}$, as first introduced in [5], are defined as follows: for an execution $\chi$ that is Zeno, $\chi$ is

Chattering Zeno: If there exists a finite $C$ such that $\tau_{i+1}-\tau_{i}=0$ for all $i \geq C$.

Genuinely Zeno: If $\tau_{i+1}-\tau_{i}>0$ for all $i \in \mathbb{N}$.

\footnotetext{
${ }^{1}$ Here we are considering only infinite executions since these are the executions that display Zeno behavior; introducing the definition of a finite execution would require unnecessary complication. For the more general definition see [14] or [15].

${ }^{2}$ In principle, other types of Zeno could be possible; for instance, when both the conditions we describe are verified infinitely often. In the paper, we consider the two extreme cases as they compliment each other and thus allow for a better understanding of Zeno behavior.
}

The difference between these two phenomena is especially prevalent in their detection and elimination. As argued in [3], Genuinely Zeno executions are much more complicated in their behavior.

\section{Sufficient CONDITIONS FOR THE EXISTENCE OF ZENO EXECUTIONS}

As mentioned in the introduction, there have been many attempts to regularize Zeno behavior by either changing the original model with the addition of some delays [15], introducing additional domains to add delays geometrically [4] and constraining the dynamics when they lie in a neighborhood of the Zeno point [7]. In [10], it was first suggested that in a very special case, a stochastic approximation of the deterministic hybrid system could effectively rule out Zeno behavior, while properly converging at the limit to the original dynamics; this idea has been reformulated and extended to a more general setting in [1].

On the detection side, the same authors in [3] have derived, for the first time, a sufficient condition for the existence of Zeno behaviors for a certain class of linear hybrid systems. After introducing the class of hybrid systems considered in the aforementioned work, we summarize the main result.

DFQ hybrid systems. A diagonal first quadrant (DFQ) hybrid system is a hybrid system of a very special form. Specifically, we say that $\mathscr{H}_{\mathbf{D F Q}}=(Q, E, D, G, R, F)$ is a DFQ hybrid system if the following further conditions hold on the set of domains, guards, reset maps and vector fields:

1) $D=\left\{D_{q}\right\}_{q \in Q}$ is, for every $q \in Q$, of the form ${ }^{3}$

$$
D_{q}=\left\{x \in \mathbb{R}^{n}: x_{1} \geq 0 \text { and } x_{2} \geq 0\right\},
$$

2) $G=\left\{G_{e}\right\}_{e \in E}$ is, for every $e \in E$, of the form

$$
G_{e}=\left\{x \in \mathbb{R}^{n}: x_{1}=0 \text { and } x_{2} \geq 0\right\},
$$

3) $R=\left\{R_{e}\right\}_{e \in E}$ is, for every $e \in E$, of the form

$$
R_{e}(x)=R_{e}\left(x_{1}, x_{2}, \ldots, x_{n}\right)=\left(x_{2}, x_{1}, \ldots, x_{n}\right)^{T},
$$

4) $F=\left\{f_{q}(x)\right\}_{q \in Q}$ is, for every $q \in Q$, of the form

$$
f_{q}(x)=\Lambda_{q} x+a_{q},
$$

where $a_{q} \in \mathbb{R}^{n}$ and $\Lambda_{q} \in \mathbb{R}^{n \times n}$ is a diagonal matrix; we denote $\left(\Lambda_{q}\right)_{i, i}$ by $\left(\lambda_{q}\right)_{i}$.

Remark 1: DFQ hybrid systems, while clearly very simple in their structure, can be used to understand general hybrid systems. In [6], it is argued that first quadrant (FQ) hybrid systems (which are only slightly more general than their DFQ counterpart) can be used to understand the stability properties of general hybrid systems by considering morphisms between these systems, i.e., "hybrid" Lyapunov functions. In this way, FQ hybrid systems are to hybrid systems as the real line is to dynamical systems - thus by understanding FQ hybrid system we can understand general hybrid systems.

\footnotetext{
${ }^{3}$ The form of these domains is the motivation for the term "first quadrant".
} 
We refer to $\Gamma=(Q, E)$ as the underlying graph of $\mathscr{H}_{\mathbf{D F Q}}$; as is intuitively obvious, it is often useful to analyze subsets of this graph. The following Theorem, proven in [3], provides sufficient conditions for the existence of Genuinely Zeno behavior in DFQ hybrid systems. The first two conditions in this theorem ensure the existence of a trajectory, while the third one is a contraction requirement.

Theorem 1: Let $\mathscr{H}_{\mathrm{DFQ}}$ be a DFQ hybrid system and $\Gamma_{\ell}=\left(Q_{\ell}, E_{\ell}\right)$ be a cycle of the underlying graph $\Gamma$ of this hybrid system. Then if $\Lambda_{q_{\ell}} x+a_{q_{\ell}}, q_{\ell} \in Q_{\ell}$, satisfies the conditions:

$$
\left.\begin{array}{c}
\left(\lambda_{q_{\ell}}\right)_{1} \leq 0 \\
\left(a_{q_{\ell}}\right)_{1}<0<\left(a_{q_{\ell}}\right)_{2} \\
\left|\prod_{q_{\ell} \in Q_{\ell}} \frac{\left(a_{q_{\ell}}\right)_{2}}{\left(a_{q_{\ell}}\right)_{1}}\right|<1
\end{array}\right\} \Rightarrow \quad \begin{gathered}
\mathscr{H}_{\mathbf{D F Q}} \text { is Genuinely } \\
\text { Zeno }
\end{gathered}
$$

\section{CONGESTION CONTROL OF COMmunication NETWORKS}

The dynamical properties of TCP schemes for the wired and wireless internet have been recently investigated by means of control theoretical techniques. Along this lines, this paper will adhere to the widespread use of the fluidflow approximation model introduced by Kelly (see [12], [13]). This dynamical model mathematically accounts for the additive increase-multiplicative decrease characteristic of the TCP on wireline networks and aptly describes its properties.

TCP model. A communication network is described via its $J$ links and its $R$ users (sender-receiver pairs). The relations between these two entities are described by a $0-1$ routing matrix $A=\left\{a_{j r}\right\}$, where $a_{j r}=1$ if user $r \in R$ uses link $j \in J$, and $a_{j r}=0$ otherwise. Each $j \in J$ has a finite capacity $C_{j}<\infty$. We model the sending rate of each user, $x_{r}(t)$ for all $r \in R$, with the following dynamical relations:

$$
\frac{d}{d t} x_{r}(t)=k_{r}\left(w_{r}^{o}-x_{r}(t) \sum_{j: a_{j r}=1} p_{j}\left(\sum_{s: a_{j s}=1} x_{s}(t)\right)\right) \text {, }
$$

where $k_{r}$ is a positive scale factor affecting the adaptation rate, and the constant $w_{r}^{o}$ can be physically interpreted as the number of connections that the user establishes with the network. The functions $p_{j}$, each specific to a single link, sum up to form the congestion signal feedback from the network to a particular user, and depend on the sum of the rates of all the users that exploit the particular link. In the wireless case, the loss functions $p_{j}$ have to account for possible non negligible channel error, along with loss due to congestion. One possible way around this problem has been described in [2], [8] and [9], where the number of connections the user establishes with the network is modified dynamically according to the observed loss functions.

In the sequel, it will be shown that a specific hybrid system (in fact, a DFQ hybrid system) can be used describe this model of a communication network by approximating the

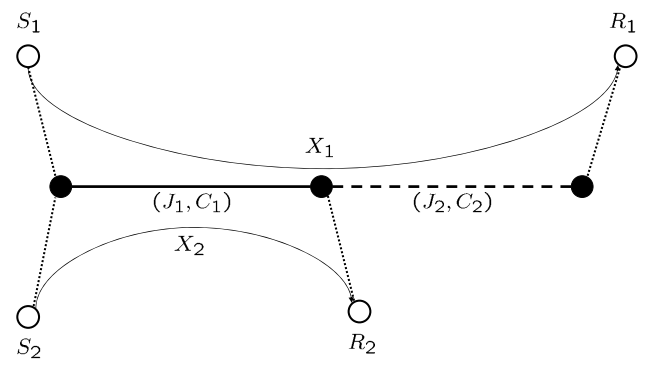

Fig. 1. The network topology for the Case Study.

nonlinear parts of its structure; within this new framework, it will be shown both analytically and a priori via the sufficient conditions given in Theorem 1, that in some instances the presence of both Chattering and Genuinely Zeno phenomena will arise. Interestingly, this relates to the presence of oscillations in steady-state in real TCP schemes.

Model for case study. We shall consider the two-users/twolinks network shown in Figure 1. The first link is assumed to be a wireline one, while the second is thought to be wireless. The model is expressed with the following pair of differential equations:

$$
\begin{aligned}
& \dot{x}_{1}=k_{1}\left(w_{1}-x_{1}\left(p_{1}\left(x_{1}+x_{2}\right)+p_{2}\left(x_{1}\right)\right)\right), \\
& \dot{x}_{2}=k_{2}\left(w_{2}-x_{2} p_{1}\left(x_{1}+x_{2}\right)\right) .
\end{aligned}
$$

It is reasonable to assume that $C_{1}>C_{2}$. The shape of the price functions $p_{j}$ determines the typology of the scheme; the only restriction on these functions is that they must be positive and increasing. In this particular instance, we shall approximate them with an indicator function centered around the congestion threshold. In order to cope with the second wireless link, according to a scheme proposed in [2], [8] and [9], the number of connections that the first user establishes, $w_{1}$, is binarily switched as follows:

$w_{1}= \begin{cases}w_{1}^{l} & \text { if }\left(x_{1}>C_{2}\right) \vee \\ & \left(\left(x_{1} \leq C_{2}\right) \wedge\left(x_{1}+x_{2} \geq C_{1}\right) \wedge\left(\dot{x}_{1} \leq 0\right)\right) \\ w_{1}^{h} \quad \text { else }\end{cases}$

It makes sense to assume that $0<w_{1}^{l}<w_{1}^{h}$. We can interpret the previous condition as follows: the first user increases the number of packets he sends through the network faster if the second link is uncongested; conversely, he decreases the number of connections-or, equivalently, additively increases the number more slowly-if the second link gets congested and as long as the first link remains clogged. Another plausible interpretation of the modifications in the value of $w_{1}$ is by a concept related to both that of "fast recovery" and that of "congestion avoidance"; the protocol tries to facilitate the decongestion of the second link by mitigating the effect of the additive-increase part of the model, while multiplicatively decreasing the congestion window rather than draining it all.

The hybrid model. The introduction of the indicator 


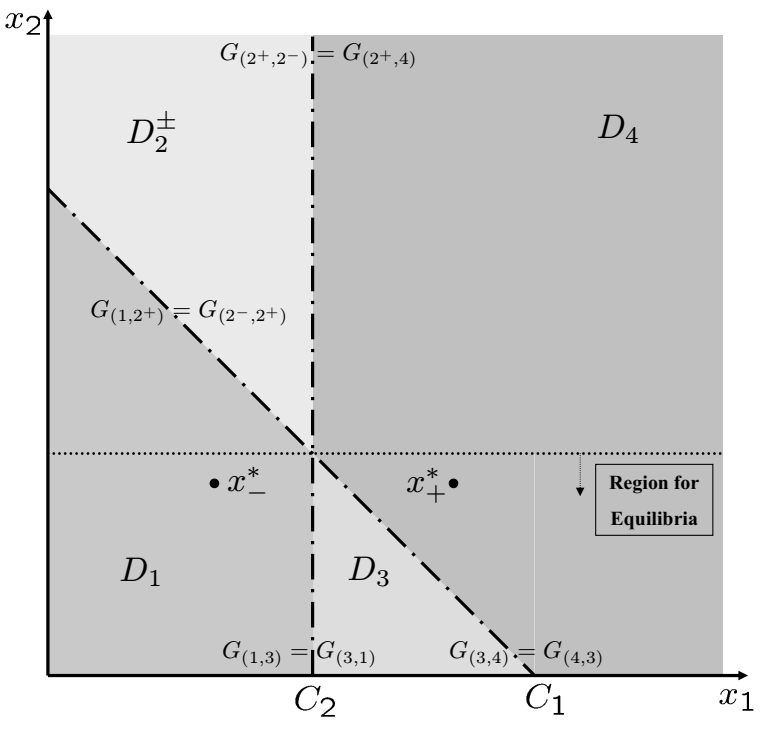

Fig. 2. The hybrid system model of the case study; the figure indicates the domains, the guards, and the region for the equilibria of the system.

function and of the discontinuity on the value of $w_{1}$ enables us to reformulate the previous model as a hybrid system. It will be composed of five modes - see Figure 2-and be specified by the following tuple:

$$
\mathscr{H}_{\mathbf{T C P}}=(Q, E, D, G, R, F),
$$

where

- $Q=\left\{1,2^{+}, 2^{-}, 3,4\right\}$,

- $E=\left\{e_{i j}=(i, j):(i, j) \in Q \times Q, i \neq j\right.$ and $(i, j) \neq$ $\left.\left(1,2^{-}\right),\left(4,2^{+}\right)\right\}$

- $D=\left\{D_{1}, D_{2}^{+}=D_{2^{+}}, D_{2}^{-}=D_{2^{-}}, D_{3}, D_{4}\right\}$, where

$$
\begin{aligned}
D_{1} & =\left\{x \in\left(\mathbb{R}_{+}^{o}\right)^{2}:\left(x_{1}+x_{2} \leq C_{1}\right) \wedge\left(x_{1} \leq C_{2}\right)\right\} \\
D_{2}^{ \pm} & =\left\{x \in\left(\mathbb{R}_{+}^{o}\right)^{2}:\left(x_{1}+x_{2} \geq C_{1}\right) \wedge\left(x_{1} \leq C_{2}\right)\right\} \\
D_{3} & =\left\{x \in\left(\mathbb{R}_{+}^{o}\right)^{2}:\left(x_{1}+x_{2} \leq C_{1}\right) \wedge\left(x_{1} \geq C_{2}\right)\right\} \\
D_{4} & =\left\{x \in\left(\mathbb{R}_{+}^{o}\right)^{2}:\left(x_{1}+x_{2} \geq C_{1}\right) \wedge\left(x_{1} \geq C_{2}\right)\right\}
\end{aligned}
$$

- $G=\left\{G_{e_{i j}}\right\}_{e_{i j} \in E}$ where $G_{e_{i j}}=\partial D_{i} \cap \partial D_{j}$, for $(i, j) \neq$ $\left(2^{+}, 2^{-}\right),\left(2^{-}, 2^{+}\right)$, with $\partial D_{i}$ the boundary of domain $D_{i}$, and

$G_{\left(2^{+}, 2^{-}\right)}=\left\{x \in\left(\mathbb{R}_{+}^{o}\right)^{2}:\left(x_{1}+x_{2} \geq C_{1}\right) \wedge\left(x_{1}=C_{2}\right)\right\}$ $G_{\left(2^{-}, 2^{+}\right)}=\left\{x \in\left(\mathbb{R}_{+}^{o}\right)^{2}:\left(x_{1}+x_{2}=C_{1}\right) \wedge\left(x_{1} \leq C_{2}\right)\right\}$

- $R=\left\{R_{e_{i j}}\right\}_{e_{i j} \in E}$ where $R_{e_{i j}}=\mathrm{Id}$, the identity map,

- $F=\left\{f_{1}, f_{2}^{+}, f_{2}^{-}, f_{3}, f_{4}\right\}$ where

$$
\begin{aligned}
f_{1}\left(x_{2}, x_{2}\right) & =\left(\begin{array}{c}
k_{1} w_{1}^{+} \\
k_{2} w_{2}
\end{array}\right) \\
f_{2}^{+}\left(x_{1}, x_{2}\right) & =\left(\begin{array}{c}
k_{1}\left(w_{1}^{+}-x_{1}\right) \\
k_{2}\left(w_{2}-x_{2}\right)
\end{array}\right) \\
f_{2}^{-}\left(x_{1}, x_{2}\right) & =\left(\begin{array}{c}
k_{1}\left(w_{1}^{-}-x_{1}\right) \\
k_{2}\left(w_{2}-x_{2}\right)
\end{array}\right) \\
f_{3}\left(x_{1}, x_{2}\right) & =\left(\begin{array}{c}
k_{1}\left(w_{1}^{-}-x_{1}\right) \\
k_{2} w_{2}
\end{array}\right) \\
f_{4}\left(x_{1}, x_{2}\right) & =\left(\begin{array}{c}
k_{1}\left(w_{1}^{-}-2 x_{1}\right) \\
k_{2}\left(w_{2}-x_{2}\right)
\end{array}\right)
\end{aligned}
$$

where here $w_{1}^{-}$and $w_{1}^{+}$can be viewed as $w_{1}^{l}$ and $w_{1}^{h}$ without the conditions on the velocity.

Qualitative dynamical analysis. Within the set of all possible hybrid trajectories, we will focus our attention at this stage on two particular subsets-those generated by initial conditions in $D_{1}$. Assume, to begin with, that the constants of the various vector fields assume values that locate the equilibria in the region shown in Figure 2. Both variables shall increase, until the intersection of the separation guard with either domain $D_{3}$ or domain $D_{2}^{+}$. In the first case, right after the event, the vector field will be pointing towards the left of the same guard. This is tantamount to chattering behavior, as shown by a simulation in Figure 3 (Bottom). The trajectory may slide on the guard and be qualitatively studied via solutions a la Filippov.

In the second scenario, which occurs along the intersection of the guards between domains $D_{1}$ and $D_{2}^{+}$, the trajectory will switch to the new mode, where it will flow towards an equilibrium point above the line $x_{1}+x_{2}=C_{1}$. This will happen until the guard between domains $D_{2}^{+}$and $D_{4}$ is hit, which induces the first user to switch to $D_{2}^{-}$and decrease the number of connections. This event will steer the trajectory towards a new equilibrium below $x_{1}+x_{2}=C_{1}$, until the first guard $G_{\left(2^{-}, 1\right)}$ will again again be intersected. Repeating this reasoning, it is possible to show that the trajectory is Zeno "by hand"-given the linear structure of the vector fields, we can solve for the trajectories with relative ease, find an explicit formula for the time steps within each domain, compute their sum and check its finiteness.

In the next section, by applying the sufficient conditions for the existence of Zeno Behavior presented in Theorem 1, we will be able to give explicit guarantees on when $\mathscr{H}_{\text {TCP }}$ is Zeno.

\section{A Priori Detection of Zeno Behavior}

In this section, we will first modify the hybrid model introduced in the previous section with the goal of transforming it into a DFQ hybrid system (Sec. III). Then, within this new framework, we shall show that the sufficient conditions for the existence of Zeno behavior apply in this particular modeling instance.

We will restrict our attention to a hybrid subsystem of the hybrid system $\mathscr{H}_{\mathbf{T C P}}$, which is represented for clarity in Figure 4. Define

$$
\mathscr{H}_{\mathbf{T C P}}^{\text {sub }}=\left(Q^{\text {sub }}, E^{\text {sub }}, D^{\text {sub }}, G^{\text {sub }}, R^{\text {sub }}, F^{\text {sub }}\right),
$$

where

- $Q^{\text {sub }}=\{+,-\}$,

- $E^{\mathrm{sub}}=\left\{e_{+}=(+,-), e_{-}=(-,+)\right\}$,

- $D^{\text {sub }}=\left\{D^{+}, D^{-}\right\}$where $D^{+}=D^{-}$are defined by the affine constraints:

$$
B x+b=\left(\begin{array}{cc}
1 & 1 \\
-1 & 0
\end{array}\right)\left(\begin{array}{l}
x_{1} \\
x_{2}
\end{array}\right)+\left(\begin{array}{c}
-C_{1} \\
+C_{2}
\end{array}\right) \geq 0
$$

Note that these constraints imply that $D^{ \pm}=D_{2}^{ \pm}$, 

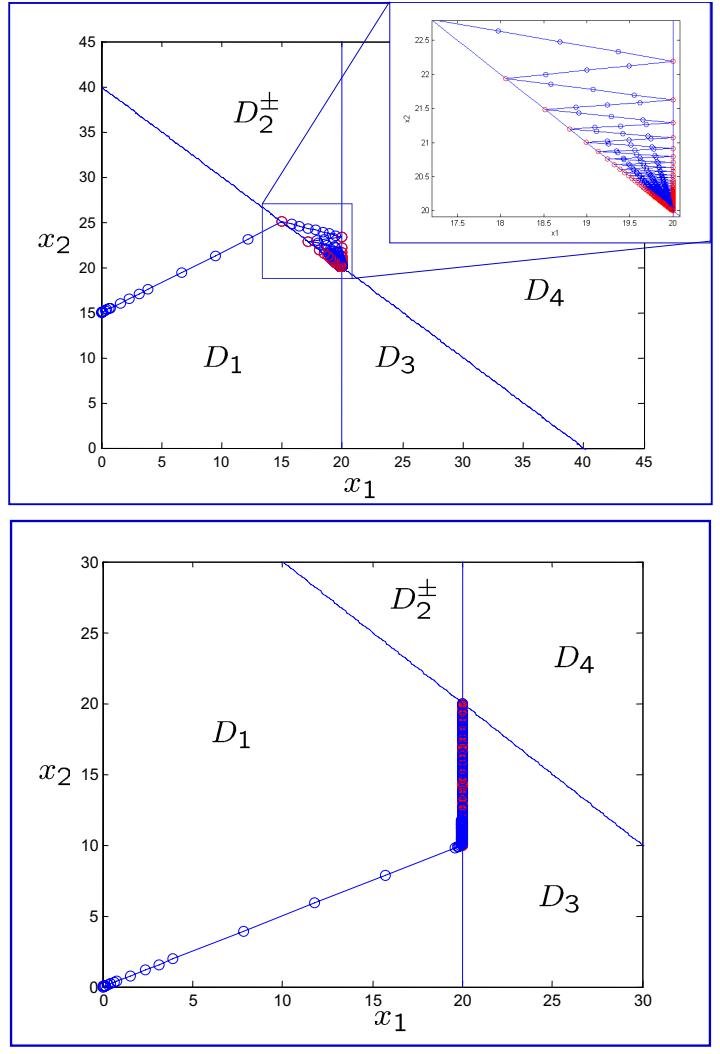

Fig. 3. Top - A hybrid trajectory presenting Genuinely Zeno behavior. Bottom - A hybrid trajectory with Chattering Zeno behavior.

- $G^{\text {sub }}=\left\{G_{e_{+}}, G_{e_{-}}\right\}$, where

$G_{e_{+}}=\left\{x \in\left(\mathbb{R}_{+}^{o}\right)^{2}:\left(x_{1}+x_{2} \geq C_{1}\right) \wedge\left(x_{1}=C_{2}\right)\right\}$

$G_{e_{-}}=\left\{x \in\left(\mathbb{R}_{+}^{o}\right)^{2}:\left(x_{1}+x_{2}=C_{1}\right) \wedge\left(x_{1} \leq C_{2}\right)\right\}$

- $R^{\text {sub }}=\left\{R_{e_{+}}, R_{e_{+}}\right\}$, where $R_{e_{ \pm}}=\mathrm{Id}$, the identity map,

- $F^{\mathrm{sub}}=\left\{f^{+}, f^{-}\right\}$, where

$$
f^{ \pm}\left(x_{1}, x_{2}\right)=-I\left(\begin{array}{c}
x_{1} \\
x_{2}
\end{array}\right)+\left(\begin{array}{c}
w_{1}^{ \pm} \\
w_{2}
\end{array}\right):=-I x+a^{ \pm}
$$

where here we have assumed ${ }^{4}$ that $k_{1}=k_{2}=1$.

The hybrid system $\mathscr{H}_{\mathbf{T C P}}^{\text {sub }}$ is important because of its ability to predict Zeno behavior in $\mathscr{H}_{\mathbf{T C P}}$, that is, we have the following (easily verifiable) lemma:

Lemma 1: If $\mathscr{H}_{\mathbf{T C P}}^{\text {sub }}$ is Genuinely Zeno, then $\mathscr{H}_{\mathbf{T C P}}$ is Genuinely Zeno.

Coordinate transformations. To transform $\mathscr{H}_{\mathrm{TCP}}^{\mathrm{sub}}$ into a DFQ hybrid system it is necessary to "bend" the guards of the domains $D^{ \pm}$to become the axes of a first quadrant, while maintaining the proper orientation of the flow. To this end, consider a dynamical system $\dot{x}=f(x)$ with $x \in \mathbb{R}^{n}$, and a diffeomorphism $\psi: \mathbb{R}^{n} \rightarrow \mathbb{R}^{n}$ that transforms the state

\footnotetext{
${ }^{4}$ In the remainder of this paper we shall make this assumption without much loss of generality.
}

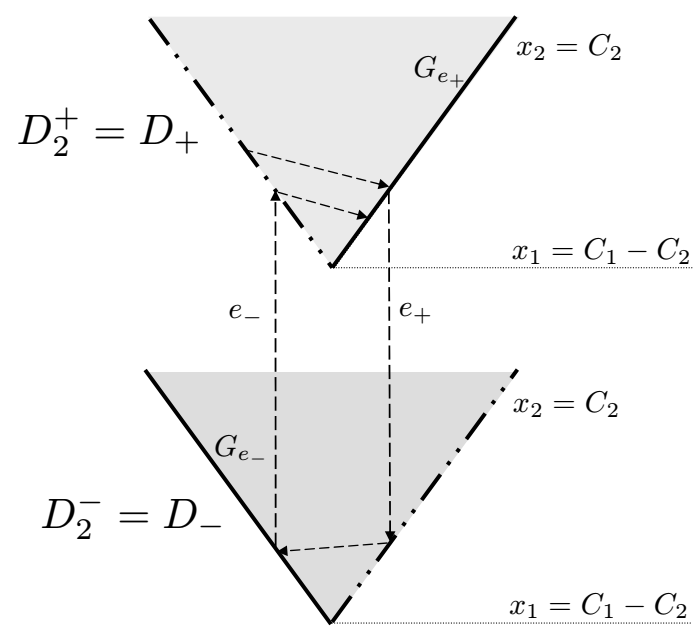

Fig. 4. The reduced hybrid system object of analysis.

space to $\widehat{x}=\psi(x)$. A solution $x(t)$ of the system will then be transformed into a solution $\widehat{x}(t)=\psi(x(t))$ of the ODE:

$$
\dot{\widehat{x}}=\frac{\partial \psi}{\partial x} f(x)=\frac{\partial \psi}{\partial x} f\left(\psi^{-1}(\widehat{x})\right)=\widehat{f}(\widehat{x}) .
$$

Note that in the case when $\psi$ is an affine transformation, i.e., $\psi(x)=H x+h$, then $\psi^{-1}(\widehat{x})=H^{-1} \widehat{x}-H^{-1} h$.

A specific transformation. There are multiple ways of defining such a diffeomorphism, but all of them should yield equivalent results when applying the sufficient conditions. We shall come up with an affine transformation $\widehat{x}=\psi(x)=H x+h$ by utilizing the affine constraints defining the domains. For both the modes, we want an affine transformation that will transform the affine constraints $B x+b \geq 0$ into the affine constraints $I \widehat{x} \geq 0$, which indeed define the first quadrant. A trivial calculation shows that it is sufficient to choose $H=B$ and $h=b$. Properly orienting the new trajectories yields the following transformations for, respectively, mode $D^{+}$and $D^{-}$:

$$
\psi^{+}(x)=\left(\begin{array}{cc}
0 & 1 \\
1 & 0
\end{array}\right) B x+b ; \quad \psi^{-}(x)=B x+b .
$$

Notice that in the first case we flip the coordinates. For the vector fields $\dot{x}=-I x+a^{ \pm}$on $D^{ \pm}$, the transformations $\psi^{ \pm}$ will yield the following vector fields:

$$
\begin{aligned}
\dot{\widehat{x}} & =\widehat{f}^{+}(\widehat{x})=-I \widehat{x}+\left(\begin{array}{cc}
0 & 1 \\
1 & 0
\end{array}\right) b+\left(\begin{array}{cc}
0 & 1 \\
1 & 0
\end{array}\right) B a^{+} ; \\
\dot{\widehat{x}} & =\widehat{f}^{-}(\widehat{x})=-I \widehat{x}+b+B a^{-} .
\end{aligned}
$$

We can now formally define a DFQ hybrid system from $\mathscr{H}_{\text {TCP }}^{\text {sub }}$ as follows:

$$
\mathscr{H}_{\mathbf{D F Q}}^{\mathbf{T C P}}=(\widehat{Q}, \widehat{E}, \widehat{D}, \widehat{G}, \widehat{R}, \widehat{F}),
$$

where $\widehat{Q}=Q^{\text {sub }}, \widehat{E}=E^{\text {sub }}$; as $\mathscr{H}_{\mathrm{DFQ}}^{\mathbf{T C P}}$ is a DFQ hybrid system we need only specific $\widehat{F}=\left\{\widehat{f}^{+}, \widehat{f}^{-}\right\}$(since the domains, guards and reset maps must be given by the specifications outlined in Sect. III) which are:

$$
\widehat{f}^{ \pm}(\widehat{x})=-I \widehat{x}+\widehat{a}^{ \pm}, \quad \text { where }
$$


$\widehat{a}^{+}=\left(\begin{array}{c}C_{2}-w_{1}^{+} \\ -C_{1}+w_{1}^{+}+w_{2}\end{array}\right) ; \widehat{a}^{-}=\left(\begin{array}{c}-C_{1}+w_{1}^{-}+w_{1} \\ C_{2}-w_{1}^{-}\end{array}\right)$

Application of the sufficient conditions. The structure of $\mathscr{H}_{\mathbf{D F Q}}^{\text {TCP }}$ allows the use of the conditions developed in [3] and summarized in Theorem 1. Because of the specific structure of $\mathscr{H}_{\mathbf{D F Q}}^{\mathbf{T C P}}$, the conditions of this theorem read:

$$
\begin{gathered}
\widehat{a}_{1}^{ \pm}<0<\widehat{a}_{2}^{ \pm} \\
\left|\frac{\widehat{a}_{2}^{+} \widehat{a}_{2}^{-}}{\widehat{a}_{1}^{+} \widehat{a}_{1}^{-}}\right|<1
\end{gathered}
$$

since the condition on the negativity of the first eigenvalue is met: $\widehat{\lambda}^{ \pm}=-1$. In other words, we have the following result, which is actually just a simple application of Theorem 1, coupled with Lemma 1.

\section{Theorem 2: $\mathscr{H}_{\mathrm{TCP}}$ is Genuinely Zeno if}

$$
\begin{gathered}
C_{2}-w_{1}^{+}<0<-C_{1}+w_{1}^{+}+w_{2} \\
-C_{1}+w_{1}^{-}+w_{2}<0<C_{2}-w_{1}^{-} \\
\left|\frac{\left(C_{2}-w_{1}^{-}\right)\left(-C_{1}+w_{1}^{+}+w_{2}\right)}{\left(C_{2}-w_{1}^{+}\right)\left(-C_{1}+w_{1}^{-}+w_{2}\right)}\right|<1
\end{gathered}
$$

To make the conditions given in this theorem slightly more transparent we assign specific values to $w_{1}^{+}, w_{1}^{-}$and $w_{2}$ based on the values of the channel capacities as follows:

$$
w_{1}^{+}=C_{2}+\alpha, \quad w_{1}^{-}=C_{2}-\alpha, \quad w_{2}=C_{1}-C_{2}-\beta,
$$

in this case, we would like to know what values of $\alpha$ and $\beta$ yield Zeno trajectories. Applying Theorem 2, we have:

\section{Corollary 1: $\mathscr{H}_{\text {TCP }}$ is Genuinely Zeno if $0<\beta<\alpha$.}

Extensions. It is possibile to extend these results. If a similar change in the number of connections is employed on the model with the original shape for the prices (which in general may be positive, smooth and increasing functions of the congestion through the specific link), it would still be possible to introduce a hybrid model of this system. We would have to deal with non-linear vector fields though, which will require results that the authors are currently investigating.

\section{CONCLUSIONS}

In this work, the sufficient conditions for the existence of Zeno trajectories in hybrid systems, as derived in [3], have been applied to the study of a special instance of wireline/wireless communication network where the flow is regulated by TCP-like protocols. We considered the fluidflow model of these protocols. The scheme was first modified to handle the errors on the wireless link followed by a simplification of its structure by the introduction of piecewise linear approximations, which allowed us to reframe the model as a hybrid system. After proper massaging, the application of the sufficient conditions proved the existence of Zeno trajectories and gave conditions under which such trajectories occur.
The result, along with being a non-trivial application of the aforementioned conditions, sheds some light on a rather important issue for TCP schemes. It is known that the introduction of approximations on the scheme, via discretizations or through the use of discontinuous functions, introduces disruptive phenomena in the system such as oscillations and chattering behavior. In this paper we showed that Zeno behavior can also be an outcome of modifications to the theoretical fluid-flow scheme. This fact advocates caution in the study of these schemes, and suggests the aid of "guarantees", both practical as well as theoretical, when dealing with these complex networks.

Acknowledgments. The first author would like to acknowledge Shams Kharimkhan for his work under the 2005 CHESS SUPERB Program at Berkeley, which provided the simulations presented in the paper.

\section{REFERENCES}

[1] A. Abate, A. Ames, and S. Sastry, "A stochastic approximation for hybrid systems," in Proceedings of the 24th American Control Conference, Portland, OR, 2005.

[2] A. Abate, M. Chen, and S. Sastry, "New congestion control schemes over wireless networks: Delay sensitivity analysis and simulations," in Proceedings of the 16th IFAC World Congress, Prague, CZ, 2005.

[3] A. D. Ames, A. Abate, and S. Sastry, "Sufficient conditions for the existence of zeno behavior," in Prooceedings of the 44th IEEE Conference on Decision and Control and European Control Conference, Seville, Spain, 2005.

[4] A. D. Ames and S. Sastry, "Blowing up affine hybrid systems." in Prooceedings of the 43rd IEEE Conference on Decision and Control, The Bahamas, 2004.

[5] — - "Characterization of zeno behavior in hybrid systems using homological methods," in Prooceedings of the 24th American Control Conference, Portland, OR, 2005.

[6] A. D. Ames, P. Tabuada, and S. Sastry, "On the stability of Zeno equilibria," in Hybrid Systems: Computation and Control, J. Hespanha and A. Tiwari, Eds., vol. LNCS 3927. Santa Barbara, CA: SpringerVerlag, 2006, pp. 34-48.

[7] A. D. Ames, H. Zheng, R. D. Gregg, and S. Sastry, "Is there life after Zeno? Taking executions past the breaking (Zeno) point," in Proceedings of the 25th American Control Conference, Minneapolis, MN, 2006.

[8] M. Chen, A. Abate, and S. Sastry, "New congestion control schemes over wireless networks: Stability analysis," in Proceedings of the 16th IFAC World Congress, Prague, CZ, 2005.

[9] M. Chen, A. Abate, A. Zakhor, and S. Sastry, "Stability and delay consideration for flow control over wireless networks," ser. Technical Report M05/14, EECS Department, UC Berkeley - ERL, 2005.

[10] J. Hespanha, "Stochastic hybrid systems: Applications to communication networks," In Rajeev Alur, George J. Pappas, Hybrid Systems: Computation and Control, vol. 2993, pp. 387-401, 2004.

[11] K. Johansson, M. Egerstedt, J. Lygeros, and S. Sastry, "On the regularization of zeno hybrid automata," Systems and Control Letters, vol. 38, pp. 141-150, 1999.

[12] F. Kelly, "Fairness and stability of end-to-end congestion control," European Journal of Control, vol. 9, pp. 159-176, 2003.

[13] F. Kelly, A. Maulloo, and D. Tan, "Rate control for communication networks: shadow prices, proportional fairness, and stability," Journal of the Operationl Research Society, vol. 49, pp. 237-252, 1998.

[14] J. Lygeros, K. Johansson, S. Simic, J. Zhang, and S. Sastry, "Dynamical properties of hybrid automata," IEEE Trans. Automat. Contr., vol. 48, no. 2-18, 2003.

[15] J. Zhang, K. H. Johansson, J. Lygeros, , and S. Sastry, "Zeno hybrid systems," International Journal of Robust and NonLinear Control, vol. 11, pp. 435-451, 2001. 